\title{
OBSTÁCULOS PARA A IMPLEMENTAÇÃO DO PROCESSO DE ENFERMAGEM NO BRASIL
}

\author{
Emilia Campos de Carvalho ${ }^{1}$ \\ Maria Márcia Bachion² \\ Maria Célia Barcellos Dalri ${ }^{3}$ \\ Cristine Alves da Costa de Jesus ${ }^{4}$
}

\section{RESUMO}

Este artigo, de reflexão teórica, busca analisar alguns fatores que têm dificultado a utilização do Processo de Enfermagem em nosso país. Resulta de um processo de aproximação, aprofundamento e de revisitação em relação à temática, que tem sido vivenciada pelas autoras nos últimos 20 anos de atuação profissional, na qual se inter-relacionam o ensino, a pesquisa, o cuidar de pessoas e a gestão da equipe de enfermagem.

Palavras chave: Processos de Enfermagem; Cuidados de Enfermagem.

\section{OBSTACLES FOR THE IMPLANTATION OF THE NURSING PROCESS IN BRAZIL}

\section{ABSTRACT}

This theoretical reflection aims to analyze some factors that have made it difficult to use the Nursing Process in our country. It is the result of an approximation, deepening and revisiting process towards the theme, which the authors have experienced in the last 20 years of their professional activities, in which teaching, research, care delivery and nursing team management are interrelated.

Keywords: Nursing Processes, Nursing Care.

\section{OBSTÁCULOS PARA IMPLEMENTAR EL PROCESO DE ENFERMERÍA EN BRASIL}

\section{RESUMEN}

Este artículo, de reflexión teórica, busca analizar algunos factores que han dificultado la utilización del Proceso de Enfermería en Brasil. Resulta de un proceso de aproximación, profundización y de revisitación con relación a la temática, que ha sido vivida por las autoras en los últimos 20 años de actuación profesional, en la cual se inter-relacionan la enseñanza, la investigación, el cuidar de personas y la gestión del equipo de enfermería.

Palabras Clave: Procesos de Enfermería, Atención de Enfermería.

${ }^{1}$ Enfermeira. Professora Titular. Docente da Escola de Enfermagem de Ribeirão Preto-Universidade de São Paulo. E-mail ecdcava@usp.br

${ }^{2}$ Enfermeira. Professora Titular. Docente do Departamento de Enfermagem. Universidade Federal de Goiás

${ }^{3}$ Enfermeira. Doutor. Docente da Escola de Enfermagem de Ribeirão Preto-Universidade de São Paulo

${ }^{4}$ Enfermeira. Doutor. Docente do Departamento de Enfermagem. Universidade de Brasília 


\section{INTRODUÇÃOO}

A organização da assistência de Enfermagem, prestada ao indivíduo, família ou comunidade, mediante utilização das fases do método científico, é denominada pela maioria dos enfermeiros no Brasil de Sistematização da Assistência de Enfermagem, também referida como SAE. Contudo, trata-se de uma terminologia que pode não se adequar ao fenômeno, uma vez que sistematizar pode significar organizar, mas não necessariamente usando todas as etapas do método científico. Assim, optamos por empregar 0 termo Processo de Enfermagem (PE).

Trata-se de uma questão semântica complexa, uma vez que, na literatura brasileira, diversas denominações são utilizadas como sinônimos ${ }^{(1)}$ : Sistematização da Assistência, Metodologia da Assistência, Planejamento da Assistência, Processo do Cuidado, Metodologia do Cuidado, Processo de Assistência, Consulta de Enfermagem, Processo de Atenção em Enfermagem e Processo de Enfermagem.

$\mathrm{Na}$ Lei $\mathrm{n}^{\circ} 7498$ de junho de 1986 que regulamenta do Exercício Profissional de Enfermagem ${ }^{(2)}$, é adotado o termo Consulta de Enfermagem, assim como na Resolução COFEN nº159 de $1993^{(3)}$; já na Resolução COFEN 272/2000 ${ }^{(4)}$ observa-se o emprego de Processo de Enfermagem.

0 Processo de Enfermagem, introduzido nos anos 50 nos Estados Unidos da América, passou por diferentes períodos evolutivos. No primeiro, quando a práxis de Enfermagem voltava sua atenção sobre a necessidade de pensar antes de agir, o PE era concebido com três fases, passando, até os anos 70, a apresentar cinco fases (assessment, diagnóstico, planejamento, implementação e avaliação). No segundo período, iniciado pelo movimento de identificação e classificação dos diagnósticos de Enfermagem, houve mudança da ênfase na identificação de problemas para ênfase no raciocínio diagnóstico. Finalmente, a partir de 1990, os focos têm sido a determinação e teste, na prática, "de resultados do paciente que sejam sensíveis à intervenção profissional"(5-7).

No Brasil, a divulgação deste método de assistência foi iniciada, na década de 70 , por Wanda de Aguiar Horta, sendo o seu impacto observado por meio de sua aplicação na assistência, no ensino e na pesquisa, até os dias atuais ${ }^{(8-11)}$.

O Conselho Internacional de Enfermagem (ICN) tem realizado esforços nos últimos anos no sentido de identificar, descrever e classificar alguns elementos que são inerentes ao processo de enfermagem: o que os exercentes da Enfermagem fazem (ações e intervenções de Enfermagem), tendo como base o julgamento sobre fenômenos humanos específicos (diagnóstico de Enfermagem), para alcançar os resultados esperados (resultados decorrentes da ação ou da intervenção de enfermagem) $)^{(12)}$.

Para aplicação do Processo de Enfermagem, é necessário um conjunto de operações cognitivas (formação de idéias, juízos e raciocínios) habilidades psicomotoras e afetivas (sentimentos, valores e crenças) além de outras competências profissionais como habilidades interpessoais ${ }^{(6)}$.

A aplicação do Processo de Enfermagem possibilita determinar os problemas que necessitam de cuidado profissional isto é aqueles pelos quais os enfermeiros são responsáveis. Embora se reconheça a importância desse Processo e tenha havido sensível mudança para sua utilização, ainda não é unânime o seu emprego no âmbito da Enfermagem ${ }^{(13-15)}$.

Este artigo, de reflexão teórica, busca apresentar alguns fatores que têm dificultado, atualmente, a utilização do PE em nosso país, bem como estratégias para enfrentá-los. Resulta de um processo de aproximação, aprofundamento e de revisitação em relação à temática, que tem sido vivenciada pelas autoras nos últimos 20 anos de atuação profissional, na qual se interrelacionam o ensino, a pesquisa, o cuidar de pessoas e a gestão da equipe de enfermagem.

- Obstáculos para utilização do PE na prática profissional e perspectivas de superação.

As dificuldades para utilização do $\mathrm{PE}$, a nosso ver, podem ser classificadas em três categorias, apresentadas a seguir, juntamente com propostas para enfrentá-las.

- Dificuldades para a utilização do PE devido a fatores inerentes a sua própria estrutura.

A complexidade do Processo de Enfermagem tem sido referida por enfermeiros e alunos dos diferentes níveis acadêmicos; em relação à abrangência da coleta de dados, da amplitude da declaração dos diagnósticos, da determinação explicita das sub-fases do planejamento (determinação de meta e objetivos) e falta de instrumentos operacionais para realizar a avaliação dos resultados alcançados mediante o cuidado prestado.

Outro aspecto diz respeito à falta de uniformidade no estabelecimento em cada uma das etapas do processo de Enfermagem. $\mathrm{O}$ fato de haver múltiplas abordagens teóricas para coleta de dados, diversas taxonomias de 
classificação da prática (diagnósticos, intervenções, resultados) têm dificultado a compreensão por parte dos profissionais, potencializando a noção de complexidade. Por outro lado, ainda há controvérsias sobre as especificidades do foco da prática de Enfermagem. Fenômenos ainda não estão claramente descritos, alguns não são consensualmente aceitos como próprios da profissão.

Para fazer frente a essas dificuldades, algumas possibilidades podem ser apontadas: aumento de investimentos em pesquisas para desvelar a especificidade e a complexidade da Enfermagem, realização de pesquisas para esclarecer as vantagens e limites do uso das classificações e modelos adotados; investigação das competências profissionais necessárias à cada etapa do $\mathrm{PE}$, estudo das limitações impostas ao PE pelo quadro clínico do paciente, da infra-estrutura dos recursos disponíveis; teste de modelos de enfermagem e etapas do processo de enfermagem para o contexto nacional.

- Dificuldades para utilização do processo de enfermagem relacionadas ao cenário de Ensino Aprendizagem

O ensino de graduação e de pós-graduação lato sensu não tem favorecido aquisição de habilidades necessárias para o desenvolvimento do Processo de Enfermagem; por outro lado, há falta de padronização do ensino de suas etapas (coleta de dados, diagnóstico, planejamento, implementação e avaliação) ao longo da formação acadêmica ${ }^{(15-16)}$.

Se considerarmos o cenário das escolas nacionais, pode-se dizer que o ensino do Processo de Enfermagem é recente, predominando a abordagem em disciplinas isoladas.

Além disso, a dinâmica de ensino não favorece a relação em tempo real entre o Processo de Enfermagem e a própria assistência de Enfermagem desenvolvida pelo aluno (os "estudos de caso" são desenvolvidos ao término do contato do aluno com 0 cliente).

As estratégias de intervenção de Enfermagem escolhidas durante o processo de formação dos alunos têm sido predominantemente restritas à realização de procedimentos, cumprimento de normas e rotinas e prática de educação em saúde, centrada no modelo bancário, não se atendo às necessidades individuais, mas às características da especialidade ou aos procedimentos a que a pessoa será submetida.

Dentre as possibilidades para redução destas dificuldades, podem ser citados: desenvolvimento de amplo projeto de Educação Permanente para profissionais docentes; utilização de estratégias que permitam a visualização dos processos cognitivos desenvolvidos pelo aluno; uso de modelos de raciocínios hipotéticos (observações, simulação, jogos, estudos de casos, situações-problema).

Além dessas, poderiam ainda ser utilizados modelos conceituais de Enfermagem para a coleta de dados, taxonomias de diagnósticos, de intervenções e de resultados de Enfermagem, com introdução precoce do exercício dos processos cognitivos necessários.

$O$ ensino do PE exige abordagem contínua ao longo da formação do aluno, que poderia ser direcionado por complexidade progressiva. Nesse sentido, a adoção do Processo de Enfermagem poderia se constituir em um eixo curricular.

A utilização de serviços que adotam Processo de Enfermagem como campo de ensino clínico, bem como a criação de redes de informações entre escolas e serviços que permitam divulgar e repetir experiências positivas poderá favorecer a consolidação de tal aprendizado.

Em situações em que não se disponha de instituições que possam ser modelos de aplicação dessa metodologia, é necessário o desenvolvimento de estratégias de ensinoaprendizagem crítico-reflexivas e transformadoras.

Finalizando, no processo de ensino dessa temática, desde o seu inicio deve ser dado enfoque ao trabalho em equipe, isto é, exercitar o aprendiz a utilizar o PE de forma integrada com o técnico e auxiliar de enfermagem. Para isso, é necessário também introduzir o ensino dessa metodologia de trabalho nos cursos Técnico e Auxiliar de Enfermagem.

\section{- Dificuldades da utilização do Processo de Enfermagem no cenário da prática assistencial}

Existe, por parte dos profissionais inseridos nas instituições de saúde, a crença de que o Processo de Enfermagem é uma "rotina sofisticada" e que executá-lo implica em "afastar-se do paciente".

Além disso, existe insegurança dos profissionais para realizar as atividades inerentes ao Processo de Enfermagem, por não dominá-las adequadamente, o que conduz à desvalorização desse método de organização do cuidar pela própria categoria.

Há, ainda, na maioria dos serviços de Enfermagem, restrição a um modelo que se volta predominantemente para a execução das ações prescritas pelo profissional médico, aliada ao fato de que na maioria das vezes o número de enfermeiros é insuficiente para o

Rev enferm UFPE on line. 2007 jul./set.; 1(1):95-9 
desenvolvimento do Processo de Enfermagem em todos os setores de atendimento aos usuários. Este quadro contribui para a falta de tempo disponível para a realização deste método de assistência no atual modelo de atendimento nas instituições.

Por outro lado, com a falta de compromisso das instituições com 0 desenvolvimento do seu capital humano, a precarização do trabalho e das condições de atendimento nas instituições de saúde, há elevado nível de incertezas no cotidiano da prática de Enfermagem: alta rotatividade de trabalhadores, alto absenteísmo, equipamentos permanentes em número insuficiente, falta de material de consumo necessário para o cuidado. Nesse sentido, é preciso revolucionar 0 modelo de gestão destas instituições.

Em decorrência das dificuldades inerentes ao processo de ensino, já descritas, encontram-se, no cenário da prática, profissionais com restrição das experiências de utilização do Processo de Enfermagem ou aplicação parcial de suas etapas.

Cabe lembrar ainda o desconhecimento do Processo de Enfermagem por parte dos técnicos e auxiliares de Enfermagem, fazendo com que não haja participação dos mesmos em sua operacionalização. Outro fator limitante nesse sentido é a falta de consenso entre os próprios enfermeiros de como pode ou deve se dar a participação desses trabalhadores na aplicação do PE.

Algumas estratégias para potencializar a utilização de tempo pelos profissionais poderiam incluir a criação de instrumentos para a coleta de dados de clientelas especificas ${ }^{(17-18)}$, a ampliação do uso da informática ${ }^{(19)}$; a revisão das atividades desenvolvidas pelo enfermeiro, definindo suas prioridades, autonomia e competências, além de mudanças na gestão que assegurem melhores condições de trabalho para cuidar dos usuários no âmbito das instituições de saúde.

É necessário ainda o estabelecimento de normas institucionais envolvendo o uso, 0 desenvolvimento e indicadores de resultados do Processo de Enfermagem, tais como utilização de taxonomias de classificação da prática, protocolos e instrumentos de avaliação; auditagem de qualidade de cuidado, determinação do custo da assistência, mensuração da satisfação de pacientes e profissionais.

A implantação de PE nas instituições de saúde é um trabalho complexo que poderia ser iniciado por unidades mais organizadas e de menor fluxo de pacientes, passando progressivamente para diferentes ambientes e a pacientes com diferentes graus de complexidade, incluindo os contextos de atenção primária, secundária e terciária à saúde. Para tanto, deve vir acompanhado de um processo de educação permanente, que favoreça a transformação do atendimento em saúde, o refinamento das habilidades e competências clínicas e do trabalho em equipe.

Nesse movimento é necessário que haja estímulo do profissional para identificar seu estilo de liderança, suas crenças sobre a natureza de Enfermagem e de sua responsabilidade para com 0 paciente/usuário.

É relevante destacar a necessidade de inclusão de todos os membros da equipe de Enfermagem em processos de educação permanente, contemplando a reflexão sobre a participação de cada um nas diferentes fases do processo de enfermagem e seus aspectos éticos e legais.

\section{CONSIDERAÇÕES FINAIS}

Foram apontadas três categorias de dificuldades para utilização do PE nos dias atuais, em nosso país; elas estão relacionadas a fatores inerentes a sua própria estrutura, ao cenário de ensino-aprendizagem e ao no cenário da prática assistencial. Diversas são as estratégias identificadas que se pode lançar mão para superá-las. Tal propósito se justifica, pois a adoção do Processo de Enfermagem enquanto método propicia uma prática reflexiva sobre as ações que estão sendo desenvolvidas pela Enfermagem na assistência.

0 uso que se fizer tanto deste método como de suas etapas, a competência que se imprimir qualidade à assistência de Enfermagem, o empenho individual e coletivo no fortalecimento da profissão, o valor atribuído ao cuidado é que poderá garantir, ou não, o avanço e a autonomia da profissão. A sua implantação dependerá das características das organizações: da política da instituição, da liderança de enfermagem e da liberdade de tomar decisões; da socialização do conhecimento sobre o tema; da comunicação existente e da vontade de se percorrer o processo de mudança na forma de assistir ${ }^{(21)}$.

\section{REFERÊNCIAS}

1. Carraro TE, Kletemberg DF, Gonçalves LM. O ensino da metodologia da assistência de Enfermagem no Paraná. Rev. Bras. de Enf. 2003; 56(5): 499-501.

2. Lei $\mathrm{n}^{\circ} 7498-$ Dispõe sobre a regulamentação do exercício da enfermagem, e dá outras providências. 1986, Publicada no Diário Oficial da União, Brasília (DF); Seção 1 de 25 de junho de 1986.

Rev enferm UFPE on line. 2007 jul./set.; 1(1):95-9 
3. Conselho Federal de Enfermagem, Resolução COFEN 159/1993. Dispõe sobre a consulta de Enfermagem. Rio de Janeiro: COFEn; 1983.

4. Conselho Federal de Enfermagem, Resolução COFEN 272/2000. Dispõe sobre Sistematização da Assistência de Enfermagem - SAE. Rio de Janeiro: COFEn; 2000.

5. Pesut DJ, Herman JA. Clinical reasoning: the art and science of critical and creative thinking. Albany: Delmar; 1999.

6. Christensen JP, Kenney JW. Nursing process: application of theories. frameworks and models 3 ed. St. Louis: Mosby; 1990.

7. Alfaro-Lefevre, R. Appliying nursing process:promoting collaborative care 5 th ed. Stuart: Lippincott; 2002.

8. Marques LVP; Carvalho DV. Sistematização da assistência de enfermagem em centro de tratamento intensivo: percepção das enfermeiras. REME. Rev. Min. Enf. 2005 jul-set; 9(3):199-205.

9. Figueiredo RM, Zen-Mascarenhas SH, Napoleão AA, Camargo AB. Caracterização da produção do conhecimento sobre sistematização da assistência de enfermagem no Brasil. Revista Escola de Enfermagem da USP 2006; 40(2): 299-303.

10. Lima, LR, Stival MM, Lima LR, Oliveira CR, Chianca TCM. Proposta de instrumento para coleta de dados de enfermagem em uma unidade de terapia intensiva fundamentado em Horta. Revista Eletrônica de Enfermagem 2006; 8(3): 349-357.

11. Cunha SMB; Barros ALB. Análise da implementação da Sistematização da Assistência de Enfermagem, segundo o Modelo Conceitual de Horta. Rev. Bras. de Enf. 2005 set-out; 58(5):568-72.

12. International Council of Nurses. The international classification for nursing practice: unifying framework - the Alpha version. Geneva: ICN; 1996.
13. Backes DS, Esperança MP, Amaro AM, Campos IEF, Cunha ADD, Schwartz ES Sistematização da assistência de Enfermagem: percepção dos enfermeiros de um hospital filantrópico. Acta Sci Health Sci 2005; 27(1):25-9.

14. Caetano JÁ, Pagliuca LMF. Autocuidado e o portador do HIV/AIDS: sistematização da assistência de Enfermagem. Rev Latino-am Enfermagem 2006 maio-junho; 14 (3):336-45.

15. Bittar DB, Pereira LV, Lemos, RCA. Sistematização da assistência de enfermagem ao paciente crítico: proposta de instrumento de coleta de dados. Texto Contexto Enferm 2006. outdez ; 15(4):617-28.

16. Agostini R. Utilização dos diagnósticos de Enfermagem - dificuldades na utilização. $1^{\circ}$ Simpósio Nacional sobre Diagnóstico de Enfermagem; 1991, nov; 19-21 Anais. São Paulo (SP):1991.

17. Carvalho EC, Garcia T. Processo de Enfermagem: o raciocínio e julgamento clínico no estabelecimento do diagnóstico de enfermagem. $3^{\circ}$ Fórum Mineiro de Enfermagem - Sistematizar o Cuidar; 2002 nov 21; Uberlândia (MG); 2002.

18. Pina JC, Mello DF, Lunardelo SR. Utilização de instrumento de registro de dados da saúde da criança e família e a prática do enfermeiro em atenção básica à saúde. Rev. Bras. Enferm. maio 2006; 59(3):270-273.

19. Hermida PMV, Araujo IEM. Elaboração e validação do instrumento de entrevista de enfermagem. Rev. Bras. Enferm. maio 2006; 59 (3):314-320.

20. Marin HF, Cunha ICKO. Perspectivas atuais da Informática em Enfermagem. Rev. Bras. Enferm. maio 2006; 59(3):354-357.

21. Maria VLR. Os caminhos do diagnóstico de enfermagem na prática de um hospital especializado. In: Guedes I, Araújo T. O uso do diagnóstico na prática de Enfermagem. Brasília: ABEn; 1997 p.77-85. Série Didática: Enfermagem no SUS.

Recebido em: 23/04/2007

Aceito em: 10/06/2007

Publicado em: 31/07/2007

Endereço para correspondência

Emilia Campos de Carvalho

Escola de Enfermagem de Ribeirão Preto/SP

Av. Bandeirantes, 3900

CEP 14040-902 - Ribeirão Preto, São Paulo, Brasil

Rev enferm UFPE on line. 2007 jul./set.; 1(1):95-9 